\title{
Do the Right Patients Use the Ambulance Service in South-Eastern Finland?
}

\author{
-A Prospective Descriptive Study of Ambulance Dispatching in Relation to the Ambulance Staff's \\ Assessment of Patients' Needs in a Subset in the South East of Finland
}

\begin{abstract}
Björn-Ove Suserud ${ }^{1}$, Lena Beillon ${ }^{2}$, Ingvar Karlberg ${ }^{3}$, Jukka Pappinen ${ }^{4}$, Maaret Castren ${ }^{5}$ Johan Herlitz ${ }^{1}$
${ }^{1}$ University of Borås, Borås, Sweden; ${ }^{2}$ Nordic School of Public Health, Göteborg, Sweden; ${ }^{3}$ Center for Health System Analysis, Gothenburg, Sweden; ${ }^{4}$ Tampere University, Tampere, Finland; ${ }^{5}$ Karolinska Institutet, Stockholm, Sweden.

Email: \{bjornove.suserud, johan.herlitz\}@hb.se, lena-marie.beillon@preem.se, ingvar.karlberg@med.gu.se, jukka.pappinen@uta.fi, maaret.castren@sodersjukhuset.se
\end{abstract}

Received September $14^{\text {th }}, 2011$; revised October 22 ${ }^{\text {nd }}, 2011$; accepted November $6^{\text {th }}, 2011$.

\begin{abstract}
Background: Several Emergency Medical Systems use a criteria-based prioritization system for ambulance response. The emergency medical priority dispatching of ambulances was introduced in the 1980s. In a system of this kind, the operators at the medical emergency dispatch centers have to assess the patients' symptoms and the need for ambulance response. The prioritization of the ambulance response is based on the seriousness of the patient's symptoms, his/her current condition and, in the case of trauma, the trauma mechanism. The priority system is supposed to optimize the use of the ambulance service and to match and meet the patients' needs with an adequate response from the ambulances. The aim of this study was to describe the dispatching and utilization of the ambulance service in a part of Finland. Results: There was a substantial divergence between the initial priority assigned and the patients' medical status at the scene. The ambulance staff confirmed the need for ambulance transport for $65 \%$ of all the patients who were assigned an ambulance by the dispatch center. Conclusions: Using a criteria-based dispatch protocol, the dispatch operator works with a wider safety margin in the priority assessments for ambulance response than was actually confirmed by the ambulance personnel at the scene. In this sample, there may be some overuse of the ambulance service. According to the assessments made by the ambulance staff, $35 \%$ of the patients did not require ambulance transport. The emergency system has to accept and work with safety margins. At the same time, there must be a balance between a safety margin and a waste of limited resources.
\end{abstract}

Keywords: Ambulance, Dispatching, Emergency Medical Services, Prioritizing

\section{Introduction}

Several Emergency Medical Systems use a criteria-based prioritization system for ambulance response. The emergency medical priority dispatching of ambulances was introduced in the 1980s. In a system of this kind, the operators at the medical emergency dispatch centers have to assess the patients' symptoms and the need for ambulance response. The prioritization of the ambulance response is based on the seriousness of the patient's symptoms, his/her current condition and, in the case of trauma, the trauma mechanism. The priority system is supposed to optimize the use of the ambulance service and to match and meet the patients' needs with an adequate response from the ambulances [1,2]. A previous study in Sweden revealed that the ambulances were dispatched for the highest priority level with blue lights and sirens for approximately one third of all the missions, but, according to the ambulance staff, only one tenth of all the patients had symptoms in relation to the priority they had been given [3]. There are ongoing discussions on the way in which criteria-based protocols optimize the use of the ambulance service [4]. It is therefore important to analyze the utilization of the EMS in relation to dispatching and the assessment of patients' symptoms, condition and the possible trauma mechanism at the scene.

The aim of this study was to describe the dispatching and utilization of the ambulance service in a part of Finland.

\section{Materials and Methods}

Finland (area 337,000 km², population 5.3 million, popula- 
tion density 17 per $\mathrm{km}^{2}$ ) is divided into 446 administratively independent municipalities, each of which is responsible for the provision of its EMS via contracts with the local fire brigade or private contractors. Health care including the EMS is publicly funded and a nominal fee is charged for ambulance transport. The national emergency telephone number, 112, connects the caller to the regional dispatch center. Currently, 15 centers are responsible for dispatching the EMS.

The dispatchers have one and a half years of formal training for handling fire, rescue and medical emergency calls. A national criteria-based dispatch system is used.

The basic ambulance level (BLS) employs fire fighters educated at the national Emergency Service College. They have 0.5 years' education in emergency care. In addition, undergraduate and registered nurses work at the basic level. The advanced level (ALS) employs nurses and paramedics with four years of high school training, but undergraduate nurses with less training were also used during the time period of this study. This means that there were no major differences between the BLS and the ALS response.

The emergency medical dispatch operators assess the incoming requests for ambulances according to a fourlevel scale. The need for an ambulance is prioritized into one of four urgency categories from A to D (Table 1).

The prioritization is based on the seriousness of the patient's chief complaint, on the patient's current condition and the trauma mechanism. Dispatching is criteria based and computer aided.

\subsection{Data Collection}

A prospective, cross-sectional study was conducted in the fall of 2006 in Finland during a three-week study period. Two ambulance districts were included in the study. The first ambulance service served about 50,000 people, population density of 70 per $\mathrm{km}^{2}$, while the second ambulance service served around 20,000 people, population density of 15 per $\mathrm{km}^{2}$. In all, the ambulance service has 7000 missions a year. The ambulance services in the studied districts have a total of four ambulances.

An expert panel formulated a specific questionnaire which was used for data collection. The ambulance staff were given both verbal and written information about the study and how to carry out the data collection. The questionnaire was then distributed to the enrolled ambulance services and the completion of one questionnaire was required for each ambulance mission, i.e. one per patient during the study period. On arriving at the patient, the ambulance crew assessed the patient's symptoms and medical condition and completed the questionnaire according to their assessment after each mission was fin- ished. All the patients during the study period were included, so no patients were excluded. The completion of one questionnaire was required for each ambulance assignment, i.e. one per patient, during the study period.

\subsection{Analysis}

For analysis, the patients were divided into groups. Chief complaints/symptoms were categorized into different main categories for data from the dispatching process and from the ambulance staff's assessment at the scene. All patients, independent of age, were registered in the study.

\section{Results}

In all, 412 patients were included in the survey. For a few of the ambulance missions included in this analysis, the information was incomplete, as indicated in the tables. There is a small variation in the number of patients in the tables due to various selection criteria.

Most of the ambulance missions (64\%) were performed between 6 a.m. and 6 p.m. Of all the patients who were assigned an ambulance, 45\% were more than 66 years of age.

People over 66 years were less frequently assigned an ambulance during the night, 12 p.m.-6 a.m. Of all the patients who were assigned an ambulance during the night, $46 \%$ were 44 years of age or younger (Table 2 ).

Table 1. Medical priority categories of ambulance response in Finland.

\begin{tabular}{cccc}
\hline Category & $\begin{array}{c}\text { Approximate } \\
\text { response time } \\
\text { (minutes) }\end{array}$ & Timing of dispatch & $\begin{array}{c}\text { Blue lights } \\
\text { and sirens }\end{array}$ \\
\hline A & 8 & Immediately & Yes \\
B & 8 & Immediately & Yes \\
C & 30 & Immediately & No \\
D & 90 & $\begin{array}{c}\text { When a suitable } \\
\text { ambulance is available }\end{array}$ & No \\
\hline
\end{tabular}

Table 2. Distribution of patients transported in an ambulance in relation to time of day and age $(n=412)$.

\begin{tabular}{ccccc}
$\begin{array}{c}\text { Age } \\
\text { categories }\end{array}$ & $\%$ & $\begin{array}{c}\text { 6 a.m.-6 p.m. } \\
\%\end{array}$ & $\begin{array}{c}\text { 6 p.m.-12 } \\
\%\end{array}$ & $\begin{array}{c}\text { p.m. } \\
\%\end{array}$ \\
\hline$<16$ & 7 & 56 & 28 & 16 \\
$17-44$ & 17 & 44 & 26 & 30 \\
$45-65$ & 30 & 61 & 26 & 13 \\
$66-80$ & 17 & 69 & 23 & 8 \\
$>81$ & 29 & 79 & 14 & 7 \\
Total & & 64 & 22 & 14 \\
\hline
\end{tabular}


Most of the calls to the dispatch center requesting an ambulance were made by persons other than the patients themselves (82\%). Slightly more than half of all the incoming calls to the emergency medical dispatch center (52\%) were made by family members or bystanders. Health care personnel made the request for an ambulance in $28 \%$ of all the cases. This means that, in $18 \%$ of all the calls, the dispatch operators spoke with the patients themselves (Table 3).

Of all the ambulance missions during the night (12 p.m.-6 a.m.), a large percentage (66\%) were prioritized by the dispatch operators as high-priority missions categories A and B.

During the day (6 a.m.-6 p.m.) and in the evening (6 p.m.-12 p.m.), the percentage of lower priority missions was higher than during the night (Table 4)

Table 3. Distribution of patients transported in ambulances in relation to who made the request for an ambulance $(n=$ 412).

\begin{tabular}{cc}
\hline $\begin{array}{l}\text { Who made the request for an } \\
\text { ambulance? } \\
(\mathrm{n}=412)\end{array}$ & $\begin{array}{c}\text { Distribution of patients } \\
\%\end{array}$ \\
\hline Patients themselves & 18 \\
Another person in family & 30 \\
Medical personnel & 28 \\
Municipal social sector personnel & 2 \\
Bystander & 22 \\
\hline
\end{tabular}

Table 4. Distribution of ambulance missions during time of day in relation to initial priority level as assessed by the emergency medical dispatchers $(n=412)$.

\begin{tabular}{|c|c|c|c|c|c|c|c|c|}
\hline \multicolumn{3}{|c|}{$\begin{array}{c}\text { Time of day } \\
6 \text { a.m. - } 6 \text { p.m. } \\
(\mathrm{n}=259)\end{array}$} & \multicolumn{3}{|c|}{$\begin{array}{c}\text { Time of day } \\
6 \text { p.m. - } 12 \text { p.m. } \\
(\mathrm{n}=100)\end{array}$} & \multicolumn{3}{|c|}{$\begin{array}{c}\text { Time of day } \\
12 \text { p.m. }-6 \text { a.m. } \\
\text { (n= }=53)\end{array}$} \\
\hline \multicolumn{3}{|c|}{$\begin{array}{l}\text { Assessment by the } \\
\text { dispatchers }\end{array}$} & \multicolumn{3}{|c|}{$\begin{array}{l}\text { Assessment by the } \\
\text { dispatchers }\end{array}$} & \multicolumn{3}{|c|}{$\begin{array}{l}\text { Assessment by the } \\
\text { dispatchers }\end{array}$} \\
\hline Priority & $\%$ & (n) & Priority & $\%$ & (n) & Priority & $\%$ & (n) \\
\hline A & 12 & (31) & A & 16 & (16) & A & 17 & (9) \\
\hline B & 31 & (80) & B & 29 & (29) & B & 49 & (26) \\
\hline C & 30 & (79) & C & 32 & (32) & C & 28 & (15) \\
\hline D & 27 & (69) & D & 23 & (23) & D & 6 & (3) \\
\hline
\end{tabular}

The dispatchers assessed $46 \%$ of all the patients as an immediate response with blue lights and sirens (category A + B). According to the ambulance crews' assessments of the patient's chief complaints and medical condition at the scene, only $8 \%$ of the patients had symptoms related to no signs of life or potentially life-threatening conditions. Most of the patients had symptoms related to neither life-threatening conditions nor a need for acute care. The ambulance staff did not confirm the need for ambulance transport for $35 \%$ of the patients who were assigned an ambulance by the dispatch center. Patients assessed and prioritized by the dispatch operators as priority $\mathrm{C}$ and $\mathrm{D}$ were more frequently assessed by the ambulance staff as not being in need of an ambulance (Table 5).

The most frequent reasons for the emergency medical dispatch operators' decisions to send out an ambulance were transport ordered by health care personnel (18\%), followed by trauma and accidents (16\%) and reduced general condition (14\%). However, of the transport ordered by health care personnel, $27 \%$ of the patients were assessed by the ambulance staff as not being in need of ambulance transport. For the patients who were assigned an ambulance for reduced general condition, slightly fewer than half (47\%) were assessed by the ambulance staff as not being in need of ambulance transport. In the trauma and accident group, 31\% of the patients were not in need of ambulance transport according to the ambu-

Table 5. Dispatch centers' initial priority level for dispatching ambulances in relation to the ambulance staff's assessment at the scene $(n=412)$.

\begin{tabular}{|c|c|c|c|c|c|c|}
\hline \multicolumn{2}{|c|}{$\begin{array}{c}\text { Dispatch } \\
\text { centers’ initial } \\
\text { priority level } \\
(\mathrm{n}=412)\end{array}$} & \multicolumn{5}{|c|}{$\begin{array}{l}\text { Patients' medical status } \\
\text { as assessed by the } \\
\text { ambulance staff } 1,2,3^{*} \\
\text { In need of an ambulance }\end{array}$} \\
\hline & & 1 & 2 & 3 & Yes & No \\
\hline Priority & $\%$ & $\%$ & $\%$ & $\%$ & $\%$ & $\%$ \\
\hline A & 13 & 40 & 41 & 19 & 80 & 20 \\
\hline B & 33 & 8 & 53 & 39 & 68 & 32 \\
\hline C & 31 & 2 & 34 & 64 & 69 & 31 \\
\hline \multirow[t]{8}{*}{ D } & 23 & 0 & 9 & 91 & 49 & 51 \\
\hline & & \multicolumn{5}{|c|}{$\begin{array}{l}\text { The ambulance staff's assessment of patients' } \\
\text { medical status at the scene: }\end{array}$} \\
\hline & & 1 & Total: & $8 \%$ & & \\
\hline & & 2 & Total: & $35 \%$ & & \\
\hline & & 3 & Total: & $56 \%$ & & \\
\hline & & \multicolumn{5}{|c|}{$\begin{array}{l}\text { Percentage of all the patients assessed by the } \\
\text { ambulance staff as being in need of }\end{array}$} \\
\hline & & \multicolumn{3}{|c|}{ an ambulance: } & Yes & No $35 \%$ \\
\hline & & & & & $65 \%$ & \\
\hline
\end{tabular}

*Patients' medical status; 1. No signs of life/potentially life-threatening condition; 2. Not life-threatening condition but in need of acute care; 3 . Neither life threatening nor in need of acute care. 
lance staff. Of all the patients who were assigned an ambulance by the dispatch center. The ambulance staff confirmed the need for ambulance transport for only 65\%. For 35\% (141/406) of all the patients, there was no need for ambulance transport according to the ambulance staff (Table 6).

According to the ambulance staff, $8 \%$ of all the patients had no signs of life or potentially life-threatening conditions when assessed at the scene. More than half of all the patients transported by the ambulance service had medical conditions assessed by the ambulance staff as neither life-threatening conditions nor in need of acute care. Of all the patients who were assigned an ambulance, $5 \%$ were assessed by the ambulance staff as treat-andleave cases. The most common reason for treat-and-leave cases was patients suffering from diabetes. The percentage of patients for whom ambulances were sent when the patients refused to be transported was $3 \%$ of all the missions. The most common reason for ambulance assignment in this category was trauma and accidents.

Table 6. Main complaints reported by the emergency medical dispatch center to the ambulance staff in relation to need for ambulance transport assessed at the scene $(n=406)$.

\begin{tabular}{|c|c|c|c|}
\hline \multirow[t]{3}{*}{$\begin{array}{l}\text { Reason for ambulance } \\
\text { assignment }(n=406) \text {. }\end{array}$} & \multirow{3}{*}{$\begin{array}{c}\begin{array}{c}\text { Overall } \\
\text { percentage }\end{array} \\
\\
\text { (\%) }\end{array}$} & \multicolumn{2}{|c|}{$\begin{array}{l}\text { Need for an ambulance } \\
\text { according to the ambu- } \\
\text { lance staff }\end{array}$} \\
\hline & & $\begin{array}{c}\text { Yes } \\
(n=265)\end{array}$ & $\begin{array}{c}\text { No } \\
(\mathrm{n}=141)\end{array}$ \\
\hline & & $65 \%$ & $35 \%$ \\
\hline $\begin{array}{l}\text { Transport ordered by health } \\
\text { care personnel }\end{array}$ & 18 & 73 & 27 \\
\hline Trauma/accidents & 16 & 69 & 31 \\
\hline Reduced general condition & 14 & 53 & 47 \\
\hline Chest pain/heart symptoms & 7 & 60 & 40 \\
\hline Abdominal symptoms & 7 & 63 & 37 \\
\hline Breathing problems & 6 & 88 & 18 \\
\hline Unconsciousness & 5 & 57 & 43 \\
\hline Psychiatric symptoms & 5 & 38 & 62 \\
\hline Intoxication & 4 & 59 & 41 \\
\hline Seizure & 3 & 83 & 17 \\
\hline Dizziness & 2 & 36 & 64 \\
\hline Unidentified need & 2 & 80 & 20 \\
\hline Extremity injuries & 2 & 71 & 29 \\
\hline Violence & 2 & 67 & 33 \\
\hline Diabetic & 1 & 100 & 0 \\
\hline Various symptoms & 1 & 25 & 75 \\
\hline Allergic symptoms & 1 & 100 & 0 \\
\hline Stroke & 1 & 67 & 33 \\
\hline Gynecological symptoms & 1 & 100 & 0 \\
\hline Headache & 1 & 100 & 0 \\
\hline
\end{tabular}

\section{Discussion}

The main findings in the study were:

- Slightly less than half of all the patients who were assigned an ambulance were more than 66 years of age.

- Most of the calls to the dispatch center requesting an ambulance were made by persons other than the patients themselves (82\%).

- The dispatchers assessed $46 \%$ of all the patients as being in need of an immediate ambulance response with blue lights and sirens.

- According to the ambulance staff, only $8 \%$ of the patients had symptoms related to no signs of life, or potentially life-threatening conditions.

- The ambulance staff confirmed the need for ambulance transport for only $65 \%$ of all the patients who were assigned an ambulance by the dispatch center.

The ambulance service in Finland is responsible for the 112 service relating to health care. As such, it provides prehospital care and the transport of medical emergencies following a 112 emergency ambulance call. To prioritize and allocate ambulances, the emergency medical dispatchers use a criteria-based dispatch protocol. A priority dispatch system should potentially enable [1]

- Faster responses to calls relating to patients with life-threatening conditions.

- Care to start as soon as a call is received.

- Lights and sirens to be used in fewer cases.

\subsection{Association between the Dispatchers' and the Ambulance Staff's Assessments}

In the study, there was a substantial divergence between the initial priority assigned and the patients' medical status at the scene. In the protocol, the ambulance crew assessed the patient's medical status at the scene and this was then compared with the decision to allocate an ambulance made by the operators at the emergency medical dispatch centers. The results showed agreement between the ambulance staff's assessment of the patient's status and the dispatcher's assessment for initial priorities in 38\% of the cases. For priority A and B, the ambulances are supposed to be allocated to patients with blue light and sirens ( $46 \%$ of the cases as judged by the dispatchers).

When the ambulance staff arrived at the scene, only $8 \%$ of all the patients were assessed as having lifethreatening conditions or being without signs of life.

The dispatchers use wider definitions for higher priority assessments for ambulance response than were actually confirmed by the ambulance staff at the scene. It is therefore possible to assume that this study shows that many of the patients in this sample could be regarded as “over-triaged". Michael and Sporer showed that dispatch protocols tend to err on the side of sensitivity at the ex- 
pense of specificity, resulting in over-triage and potential overtreatment by prehospital providers [5]. The emergency medical dispatcher's limited decision time may mean that an overestimation of safety margins for the highest priority level is be expected. The challenge is to strike a balance between over-triage/under-triage and the risk of, on the one hand, the overuse of limited resources and, on the other hand, missing cases.

In one Swedish study, the authors argue in favor of recurrent feedback to the operators and further evaluation of the protocol used by dispatch centers, the handling of incoming calls and the interviews [6]. These could be a way of increasing the accuracy of optimal initial prioritization. Another alternative is to increase the dispatcher's decision time, at least in some cases. However, there are cases, such as a presumed cardiac arrest, where the decision time must remain very short.

\subsection{The Need for an Ambulance among Transported Patients}

The ambulance staff assessed that only $65 \%$ of all patients in this sample actually required ambulance transport. Transporting patients without the need for care could be regarded as the ineffective use of trained emergency personnel and advanced equipment. It is therefore important to optimize the use of the ambulance service to match the demand for cost efficiency. If our results are good enough to be generalized, slightly more than about one third of all patients for whom an ambulance is dispatched are not in need of ambulance transport. This is a large number of patients in a system with limited resources.

According to Langhelle and colleagues, the role of prehospital emergency care in Finland is growing because of the closure of smaller hospitals during out-ofoffice hours, thereby increasing transport times from the scene to definitive care [7]. This will lead to longer transport for ambulances and an increased need of back-up and it is therefore even more important that the EMS service is used optimally. With several unnecessary transports, the need for additional back-up units increases.

In the future, research activities should focus on studies which evaluate alternative treatments for patients not judged as requiring transport by ambulance to emergency departments.

It might be difficult for the dispatchers to make an assessment of this kind.

\subsection{Dispatch and Use of the Ambulance Service in Finland and Sweden}

According to Langhelle and co-workers, the Nordic Emergency Medical Service (EMS) systems have more similarities than differences [7].
For example, in Finland and Sweden, the dispatch of ambulances is performed by dispatch centers via the national emergency phone number, 112. Both countries use a criteria-based dispatch system from which the dispatch operators assess the patients' need for ambulance response. When comparing this study of the dispatch and use of the ambulance service in Finland and an equivalent geographical area in Sweden [8], we found both similarities and differences of interest.

- In both countries, it is the older patients that most frequently use the EMS.

- Over-triage and safety margins for initial priority settings were found in both Finland and Sweden.

\subsection{Strengths and Limitations}

The strengths of this study are the prospective design and the consecutive enrolment of data gathered over a short period of time. The limitations are that the study cannot be regarded as entirely representative, due to the small sample size and the fact that only a small part of Finland was included. Another limitation is associated with the lack of accepted criteria for prehospital needs assessment. In this study, the emergency medical dispatchers used their routine guidelines and the ambulance staff made the needs assessments based on the protocol developed for the study. Furthermore, another limitation is that we did not listen to the calls to the emergency medical dispatch center to find out whether the guidelines were followed by every dispatcher.

An additional limitation is that a good evaluation of ambulance assignments should probably include an audit of the 112 calls themselves and the information available to the dispatchers. Another limitation is the absence of data on the in-hospital phase for the enrolled patients.

\section{Conclusions}

Using a criteria-based dispatch protocol, the dispatch operator works with a wider safety margin in the priority assessments for ambulance response than was actually confirmed by the ambulance personnel at the scene. In this sample, there may be some overuse of the ambulance service. According to the assessments made by the ambulance staff, $35 \%$ of the patients did not require ambulance transport. The emergency system has to accept and work with safety margins. At the same time, there must be a balance between a safety margin and a waste of limited resources. A worrying number of calls come from health care professionals. This might reflect a lack of knowledge of how the EMS in their community works and what the real resources of the local system are.

In the future, research activities need to focus on decision support systems and feedback systems for the dispatchers in order to increase the accuracy of their priori- 
tization.

Furthermore, alternative treatments must be developed for patients who do not fulfill the criteria for ambulance transport to the emergency department.

\section{REFERENCES}

[1] J. J. Clawson, "Hauert Dispaching Life Support-Establishing Standards That Work," Journal of Emergency Medicine Service, Vol. 154, 1990, pp. 82-88.

[2] L. L. Culley, D. K. Henwood, J. J. Clark, M. S. Eisenberg and C. Horton, "Increasing the Efficiency of Emergency Medical Services by Using Criteria Based Dispatch," Annual of Emergency Medicine, Vol. 24, No. 5, 1994, pp. 867-872. doi:10.1016/S0196-0644(54)00223-5

[3] L. Hjälte, B. O. Suserud, J. Herlitz and I. Karlberg, "Initial Emergency Medical Dispatching and Prehospital Need Assessment. A Prospective Study of the Swedish Ambulance Service," European Journal of Emergency Medicine, Vol. 14, No. 3, 2007, pp. 134-156. doi:10.1097/MEJ.0b013e32801464cf

[4] M. W. Cooke, "The Use of Criteria Based Dispatch in the Prioritisation of 999 Emergency Ambulance Calls,” De- partment of Primary Care \& General Practice, Faculty of Medicine, The University of Birmingham, Birmingham, 2001.

[5] G. E. Michael and K. A. Sporer, "Validation of Low-Acuity Emergency Medical Services Dispatch Codes,” Prehospital Emergency Care, Vol. 9, No. 4, 2005, pp. 429-433. doi:10.1080/10903120500255875

[6] A. Bang, P. O. Ortgren, J. Herlitz and P. Warborg, "Dispatcher-Assisted Telephone CPR: A Qualitative Study Exploring How Dispatchers Perceive Their Experience," Resuscitation, Vol. 53, No. 2, 2002, pp. 135-151. doi:10.1016/S0300-9572(01)00508-1

[7] A. Langhelle, H. M. Lossius, T. Silfvast, H. M. Björnsson, F. Lippert, A. Ersson and E. Söreide, "International EMS System: The Nordic Countries,” Resuscitation, Vol. 61, No. 1, 2004, pp. 9-21. doi:10.1016/j.resuscitation.2003.12.008

[8] L. M. Beillon, B. O. Suserud, I. Karlberg and J. Herlitz, "Does Ambulance Use Differ between Geographic Areas? A Survey of Ambulance Use in Sparsely and Densely Populated Areas," The American Journal of Emergency Medicine, Vol. 27, No. 2, 2009, pp. 202-211. doi:10.1016/j.ajem.2008.01.012 